JOURNAL OF

SYMPLECTIC GEOMETRY

Volume 5, Number 2, 139-166, 2007

\title{
A COMPACT SYMPLECTIC FOUR-MANIFOLD ADMITS ONLY FINITELY MANY INEQUIVALENT TORIC ACTIONS
}

\author{
Yael Karshon, Liat Kessler, and Martin Pinsonnault \\ Let $(M, \omega)$ be a four-dimensional compact connected symplectic \\ manifold. We prove that $(M, \omega)$ admits only finitely many inequivalent \\ Hamiltonian effective 2-torus actions. Consequently, if $M$ is simply \\ connected, the number of conjugacy classes of 2-tori in the symplecto- \\ morphism group $\operatorname{Sympl}(M, \omega)$ is finite. Our proof is "soft". The proof \\ uses the fact that if a symplectic four-manifold admits a toric action, \\ then the restriction of the period map to the set of exceptional homol- \\ ogy classes is proper. In an appendix, we present the Gromov-McDuff \\ properness result for a general compact symplectic four-manifold.
}

Dedicated to Dusa McDuff

\section{Introduction}

An action of the torus $\mathbb{T}^{k}=\left(S^{1}\right)^{k}$ on a symplectic manifold $(M, \omega)$ is a homomorphism

$$
\rho: \mathbb{T}^{k} \rightarrow \operatorname{Sympl}(M, \omega)
$$

from the torus to the group of symplectomorphisms such that the map $\rho^{\dagger}: \mathbb{T}^{k} \times M \rightarrow M$ given by $(a, m) \mapsto \rho(a)(m)$ is smooth. A sub-torus of $\operatorname{Sympl}(M, \omega)$ is the image of such a homomorphism. We restrict our attention to actions that are effective (faithful: $\rho$ is one-to-one).

We say that two actions $\rho_{1}: \mathbb{T}^{k} \rightarrow \operatorname{Sympl}(M, \omega)$ and $\rho_{2}: \mathbb{T}^{k} \rightarrow \operatorname{Sympl}(M, \omega)$ are equivalent if there exists a symplectomorphism $\psi: M \rightarrow M$ and an automorphism $h: \mathbb{T}^{k} \rightarrow \mathbb{T}^{k}$ such that the diagram

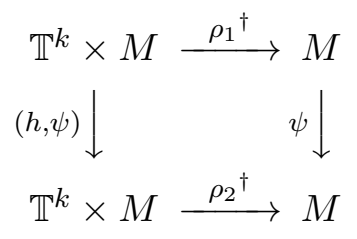


commutes. Two (effective) torus actions on $(M, \omega)$ are equivalent if and only if their images are conjugate sub-tori of $\operatorname{Sympl}(M, \omega)$.

A $\mathbb{T}^{k}$-action on $(M, \omega)$ with generating vector fields $\xi_{1}, \ldots, \xi_{k}$ is Hamiltonian if it has a moment map, that is, a map $\Phi: M \rightarrow \mathbb{R}^{k}$ whose components satisfy Hamilton's equation $d \Phi_{j}=-\iota\left(\xi_{j}\right) \omega$. If $H^{1}(M ; \mathbb{R})=\{0\}$ then every torus action on $(M, \omega)$ is Hamiltonian. An (effective) Hamiltonian torus action is toric if the dimension of the torus is half the dimension of the manifold; if $M$ is compact and connected, the triple $(M, \omega, \Phi)$ is called a symplectic toric manifold. In this paper we prove the following "finiteness theorem".

Theorem 1.1. Let $(M, \omega)$ be a four-dimensional compact connected symplectic manifold. Then the set of equivalence classes of toric actions on $(M, \omega)$ is finite.

An important ingredient in the proof of Theorem 1.1 is the following "properness lemma".

Lemma 1.2. Let $(M, \omega)$ be a symplectic four-manifold with $b_{2}^{+}=1$. Then the restriction of the period map $E \mapsto \omega(E)$ to the set of classes in $H_{2}(M ; \mathbb{R})$ with self intersection -1 is proper.

We prove Lemma 1.2 and Theorem 1.1 by "soft" equivariant, algebraic, and combinatorial techniques. Our first proof used "hard" holomorphic techniques, and relied on a "properness lemma", due to Gromov and McDuff, that applies to general compact symplectic four-manifolds. This earlier approach, although it used stronger machinery than necessary, is still worth presenting, for a number of reasons. First, this approach exhibits the interplay between "soft" and "hard" techniques. (See [19] for another usage of holomorphic techniques in proving results on toric actions.) Second, as far as we know, now these holomorphic techniques are necessary for generalizing our "finiteness" results to actions of other groups [33]. Finally, this volume in honour of Dusa McDuff provides an opportunity to present the general Gromov-McDuff properness lemma in a form that should be useful for non-experts. We present this holomorphic approach in an appendix.

Theorem 1.1 has the following corollary.

Corollary 1.3. Let $(M, \omega)$ be a four-dimensional compact connected symplectic manifold with $H^{1}(M ; \mathbb{R})=\{0\}$. Then the number of conjugacy classes of 2-tori in $\operatorname{Sympl}(M, \omega)$ is finite.

The number of conjugacy classes can be arbitrarily large; see Example 2.6. If $(M, \omega)$ admits a toric action then $M$ is simply connected [15, Corollary $32.2]$, so $H^{1}(M ; \mathbb{R})=\{0\}$. We restrict our attention to symplectic manifolds $(M, \omega)$ such that $M$ is compact and connected and $H^{1}(M ; \mathbb{R})=\{0\}$ and, as before, to torus actions that are effective. 
Since the orbits of a Hamiltonian torus action are isotropic (see [4, Proposition III.2.12]) and, on an open dense set, the (effective) action is free (see [11, Corollary B.48]), a toric action does not extend to a Hamiltonian action of a larger torus. Because $H^{1}(M, \mathbb{R})=0$, every symplectic torus action is Hamiltonian. Thus, the image of a toric action is a maximal torus in the symplectomorphism group $\operatorname{Sympl}(M, \omega)$. If $\operatorname{dim} M=4, H^{1}(M ; \mathbb{R})=\{0\}$, and $\operatorname{dim} H^{2}(M ; \mathbb{R}) \leq 3$, every Hamiltonian $S^{1}$-action on $(M, \omega)$ extends to a toric action [18, Theorem 1], so that in this case every maximal torus in the symplectomorphism group comes from a toric action. However, in general, there exist Hamiltonian $S^{1}$-actions that do not extend to toric actions, so there exist maximal tori that do not come from toric actions. In fact, some symplectic four-manifolds admit $S^{1}$-actions and do not admit any toric actions. For others, the symplectomorphism group contains some maximal tori that are two-dimensional and some that are one-dimensional. See Example 3.5. However, most symplectic four-manifolds do not admit any circle actions (in particular, they do not admit any toric actions); see Example 3.5, and for many more examples see $[\mathbf{1 2}]$.

Maximal tori play a central role in the theory of finite-dimensional Lie groups. In a compact Lie group, every two maximal tori are conjugate. Our work can be viewed as an attempt to find aspects of finite-dimensional Lie theory that carry over to groups of symplectomorphisms. See $[\mathbf{6}, \mathbf{2 1}, \mathbf{3 4}]$ for related ideas. Homotopy theoretic properties of symplectomorphism groups have been studied in $[\mathbf{1}, \mathbf{2}, \mathbf{7}, \mathbf{1 3}, \mathbf{2 2}, \mathbf{3 5}]$; see the survey [30].

At this point we do not know whether a finiteness result similar to Theorem 1.1 also holds in higher dimensions or for non-Hamiltonian symplectic group actions. The analogous result does not hold for contactomorphisms: Eugene Lerman [23] constructed a compact contact three-manifold that admits infinitely many non-conjugate toric actions. "Finiteness" also does not hold for the groups of all diffeomorphisms: for example, $\operatorname{Diff}\left(S^{2} \times S^{2}\right)$ contains infinitely many non-conjugate twodimensional maximal tori; see Example 2.6.

\section{Four-dimensional symplectic toric manifolds and corner chopping}

The automorphism group of the torus $\mathbb{T}^{n}=\mathbb{R}^{n} / 2 \pi \mathbb{Z}^{n}$ is the group $\operatorname{GL}(n, \mathbb{Z})$ of $n \times n$ matrices with integer entries and determinant equal to 1 or -1 . If a matrix $A$ belongs to $\operatorname{GL}(n, \mathbb{Z})$ then so do its transpose $A^{T}$ and its inverse $A^{-1}$. We also consider the group $\operatorname{AGL}(n, \mathbb{Z})$ of affine transformations of $\mathbb{R}^{n}$ that have the form $x \mapsto A x+\alpha$ with $A \in \operatorname{GL}(n, \mathbb{Z})$ and $\alpha \in \mathbb{R}^{n}$.

A Delzant polytope in $\mathbb{R}^{n}$ is a compact convex polytope $\Delta$ such that the edges emanating from each vertex can be generated by a $\mathbb{Z}$-basis of the lattice $\mathbb{Z}^{n}$. 
Remark 2.1. Other authors also use the following terms for polytopes with this property: non-singular, primitive, regular, smooth, unimodular and torsion free.

An important model for a toric action is $\mathbb{C}^{n}$ with the standard symplectic form, the standard $\mathbb{T}^{n}$-action given by rotations of the coordinates, and the moment map

$$
\left(z_{1}, \ldots, z_{n}\right) \mapsto \frac{1}{2}\left(\left|z_{1}\right|^{2}, \ldots,\left|z_{n}\right|^{2}\right) .
$$

The image of this moment map is the positive orthant,

$$
\mathbb{R}_{+}^{n}=\left\{\left(s_{1}, \ldots, s_{n}\right) \mid s_{j} \geq 0 \text { for all } j\right\} .
$$

The moment map image of a symplectic toric manifold is a Delzant polytope. A Delzant polytope can be obtained by gluing open subsets of $\mathbb{R}_{+}^{n}$ by means of elements of $\operatorname{AGL}(n, \mathbb{Z})$. Similarly, a symplectic toric manifold can be obtained by gluing open $\mathbb{T}^{n}$-invariant subsets of $\mathbb{C}^{n}$ by means of equivariant symplectomorphisms and reparametrizations of $\mathbb{T}^{n}$. In fact,

Lemma 2.2. Let $(M, \omega)$ be a symplectic toric manifold with moment map $\Phi: M \rightarrow \operatorname{Lie}\left(\mathbb{T}^{n}\right)^{*}=\mathbb{R}^{n}$. Let $x$ be a point in $\mathbb{R}^{n}$. Then there exists an open neighborhood of $x$ whose moment map preimage is equivariantly symplectomorphic to an open subset of $\mathbb{C}^{n}$ with the standard $\mathbb{T}^{n}$-action conjugated by some automorphism $\mathbb{T}^{n} \rightarrow \mathbb{T}^{n}$.

Proof. Because the moment map image is closed, the lemma is true if $\Phi^{-1}(x)$ is empty.

Let $O$ be an orbit in $\Phi^{-1}(x)$. By the local normal form for Hamiltonian torus actions, some neighborhood $U$ of $O$ is equivariantly symplectomorphic to an open subset of $\mathbb{C}^{n}$. In particular, the level set $\Phi^{-1}(x)$ is a discrete union of orbits. Because the level sets are connected $[\mathbf{3}, \mathbf{1 4}], \Phi^{-1}(x)=O$. Because $\Phi$ is proper, the inverse image of a sufficiently small neighborhood of $x$ is contained in $U$.

Delzant [8] classified symplectic toric manifolds up to equivariant symplectomorphism. Applying his theorem to different toric actions on a fixed manifold, we get the following result.

Proposition 2.3. Let $(M, \omega)$ be a $2 n$-dimensional compact connected symplectic manifold.

(1) For any Hamiltonian $\mathbb{T}^{n}$-action on $(M, \omega)$, its moment map image is a Delzant polytope in $\mathbb{R}^{n}$.

(2) Two Hamiltonian $\mathbb{T}^{n}$-actions on $(M, \omega)$ are equivalent if and only if their moment map images are $\operatorname{AGL}(n, \mathbb{Z})$-congruent.

Proof. By $[\mathbf{3}, \mathbf{1 4}]$, the moment map image is a convex polytope. Lemma 2.2 implies that this polytope is locally $\operatorname{AGL}(n, \mathbb{Z})$-congruent to $\mathbb{R}_{+}^{n}$; thus, it is a Delzant polytope. 
To prove part (2), let $\rho_{1}, \rho_{2}: \mathbb{R}^{n} \rightarrow \operatorname{Sympl}(M, \omega)$ be toric actions with moment maps $\Phi_{1}, \Phi_{2}: M \rightarrow \mathbb{R}^{n}$ and moment map images $\Delta_{1}$ and $\Delta_{2}$.

Suppose that the actions $\rho_{1}$ and $\rho_{2}$ are equivalent, i.e., there exist $A \in$ $\operatorname{GL}(n, \mathbb{Z})$ and $\psi \in \operatorname{Sympl}(M, \omega)$ such that

$$
\rho_{2}(A \cdot \lambda)=\psi \circ \rho_{1}(\lambda) \circ \psi^{-1}
$$

for all $\lambda \in \mathbb{T}^{n}$. A moment map for the $\mathbb{T}^{n}$-action $\lambda \mapsto \rho_{2}(A \cdot \lambda)$ is $A^{T} \circ \Phi_{2}$. A moment map for the $\mathbb{T}^{n}$-action $\lambda \mapsto \psi \circ \rho_{1}(\lambda) \circ \psi^{-1}$ is $\Phi_{1} \circ \psi^{-1}$. By $(2.2)$, these are moment maps for the same action, so their difference is a constant: for some $\alpha \in \mathbb{R}^{n}$,

$$
\Phi_{1} \circ \psi^{-1}=A^{T} \circ \Phi_{2}+\alpha .
$$

Comparing the images of these maps, we get $\Delta_{1}=A^{T} \Delta_{2}+\alpha$. So $\Delta_{1}$ and $\Delta_{2}$ are $\operatorname{AGL}(n, \mathbb{Z})$-congruent.

Suppose that $\Delta_{1}$ and $\Delta_{2}$ are $\operatorname{AGL}(n, \mathbb{Z})$-congruent. Let $A \in \operatorname{GL}(n, \mathbb{Z})$ and $\alpha \in \mathbb{R}^{n}$ be such that

$$
\Delta_{2}=A \Delta_{1}+\alpha
$$

The map $A \circ \Phi_{1}+\alpha$ is a moment map for the action $\rho_{1} \circ A^{T}$. Its image, $A \Delta_{1}+\alpha$, coincides with the image $\Delta_{2}$ of the moment map for the action $\rho_{2}$. By Delzant's theorem [8], toric actions with the same moment map image are equivariantly symplectomorphic. Applying this to the actions $\rho_{1} \circ A^{T}$ and $\rho_{2}$, we find that there exists a symplectomorphism $\psi: M \rightarrow M$ such that $\psi \circ \rho_{1}\left(A^{T} \cdot \lambda\right)=\rho_{2}(\lambda) \circ \psi$ for all $\lambda \in \mathbb{T}^{n}$. That is, the actions $\rho_{1}$ and $\rho_{2}$ are equivalent.

2.4. The rational length of an interval $d$ of rational slope in $\mathbb{R}^{n}$ is the unique number $\ell=\operatorname{length}(d)$ such that the interval is $\operatorname{AGL}(n, \mathbb{Z})$-congruent to an interval of length $\ell$ on a coordinate axis. In what follows, intervals are always measured by rational length.

Example 2.5. Figure 1 shows examples of Delzant polygons with three and four edges. On the left there is a Delzant triangle,

$$
\Delta_{\lambda}=\left\{\left(x_{1}, x_{2}\right) \mid x_{1} \geq 0, x_{2} \geq 0, x_{1}+x_{2} \leq \lambda\right\} .
$$

This is the moment map image of the standard toric action on $\mathbb{C P}^{2}$, with the Fubini-Study symplectic form normalized so that the symplectic area of $\mathbb{C P}^{1} \subset \mathbb{C P}^{2}$ is $2 \pi \lambda$. The rational lengths of all its edges is $\lambda$.

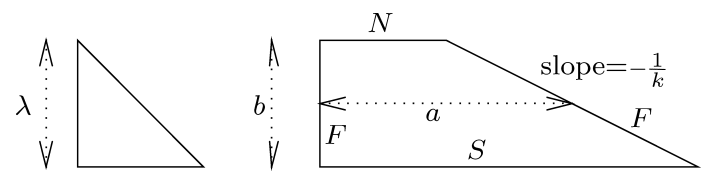

Figure 1. A Delzant triangle, $\Delta_{\lambda}$, and a Hirzebruch trapezoid, $H_{a, b, k}$. 
On the right there is a Hirzebruch trapezoid,

$$
H_{a, b, k}=\left\{\left(x_{1}, x_{2}\right) \mid-\frac{b}{2} \leq x_{2} \leq \frac{b}{2}, 0 \leq x_{1} \leq a-k x_{2}\right\},
$$

where $b$ is the height of the trapezoid, $a$ is its average width, and $k$ is a non-negative integer such that the right edge has slope $-1 / k$ or is vertical if $k=0$. We assume that $a \geq b$ and that $a-k \frac{b}{2}>0$. This trapezoid is a moment map image of a standard toric action on a Hirzebruch surface. The rational lengths of its left and right edges are $b$; the rational lengths of its top and bottom edges are $a \pm k b / 2$.

Example 2.6. Consider the manifold $S^{2} \times S^{2}$ with the product symplectic form $\omega_{a, b}=a \tau \oplus b \tau$, where $\tau$ is the rotation invariant area form on $S^{2}$ with total area $2 \pi$. For any toric action on $\left(S^{2} \times S^{2}, \omega_{a, b}\right)$, its moment map image is $\operatorname{AGL}(2, \mathbb{Z})$-congruent to the Hirzebruch trapezoid $H_{a, b, k}$ for some non-negative even integer $k$. See $[\mathbf{1 8}]$. Given $a$ and $b$, there exist only finitely many such $k$ 's for which $a-k \frac{b}{2}>0$. Hence, up to conjugation, there are only finitely many 2-tori in the symplectomorphism group of $\left(S^{2} \times S^{2}, \omega_{a, b}\right)$.

Different non-negative even integers $k$ correspond to 2-tori in the symplectomorphism group $\operatorname{Sympl}\left(S^{2} \times S^{2}, \omega_{a, b}\right)$ that are not conjugate. The 2-tori corresponding to different integers $k$ are not even conjugate in the group $\operatorname{Diff}\left(S^{2} \times S^{2}\right)$ of diffeomorphisms of $S^{2} \times S^{2}$ : the value of $k$ can be recovered from the fact that the two-dimensional orbit type strata have self intersection $0, k$, and $-k$. These tori are maximal in $\operatorname{Diff}\left(S^{2} \times S^{2}\right)$ : because an effective compact group action on a connected manifold is also effective on invariant open subsets, and by local linearization, a torus that acts effectively on a manifold with a fixed point is at most of half the dimension of the manifold. Hence, Diff $\left(S^{2} \times S^{2}\right)$ contains infinitely many conjugacy classes of maximal tori.

For each Hirzebruch trapezoid $H_{a, b, k}$ with $k$ even there exists a torus action on $\left(S^{2} \times S^{2}, \omega_{a, b}\right)$ with moment map image $H_{a, b, k}$. See [18].

2.7. Let $d, d^{\prime}$, and $d^{\prime \prime}$ be three consecutive edges in a Delzant polygon, ordered counterclockwise. Let $u, u^{\prime}$, and $u^{\prime \prime}$ be their outward normal vectors, normalized so that they are primitive lattice elements. Then each of $\left(u, u^{\prime}\right)$ and $\left(u^{\prime}, u^{\prime \prime}\right)$ is an oriented $\mathbb{Z}$-basis for $\mathbb{Z}^{2}$. It follows that there exists an integer $k$ such that $u+u^{\prime \prime}=k u^{\prime}$. We define the combinatorial self intersection number of $d^{\prime}$ to be $-k$. We also say that the combinatorial intersection number of two different edges is 1 if they are adjacent and 0 if not.

Example 2.8. The edges of the polygon in Figure 2 have the following rational lengths and combinatorial self intersections. 


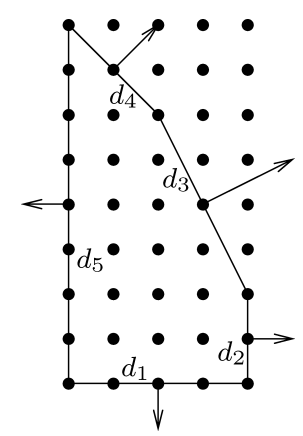

Figure 2. Polygon for Example 2.8.

\begin{tabular}{ccc}
\hline & rational length & combinatorial self intersection \\
\hline$d_{1}$ & 4 & 0 \\
$d_{2}$ & 2 & -2 \\
$d_{3}$ & 2 & -1 \\
$d_{4}$ & 2 & -1 \\
$d_{5}$ & 8 & 1 \\
\hline
\end{tabular}

2.9. An almost complex structure on a $2 n$-dimensional manifold $M$ is an automorphism of the tangent bundle, $J: T M \rightarrow T M$, such that $J^{2}=-$ id. It is tamed by a symplectic form $\omega$ if $\omega(v, J v)>0$ for all $v \neq 0$. The first Chern class of the symplectic manifold $(M, \omega)$ is defined to be the first Chern class of the complex vector bundle $(T M, J)$ and is denoted $c_{1}(T M)$. This class is independent of the choice of tamed almost complex structure $J$ $[31, \S 2.6]$.

Lemma 2.10. Let $(M, \omega)$ be a compact connected symplectic four-manifold. Let $\Phi: M \rightarrow \mathbb{R}^{n}$ be the moment map for a toric action, and let $\Delta$ be its image.

(1) Let $d$ be an edge of $\Delta$ of rational length $\ell$. Then its preimage, $\Phi^{-1}(d)$, is a symplectically embedded 2-sphere in $M$ of symplectic area

$$
\int_{\Phi^{-1}(d)} \omega=2 \pi \ell
$$

(2) Let $d$ and $d^{\prime}$ be edges of $\Delta$. Then their combinatorial intersection number (see §2.7) is equal to the intersection number of their preimages in $M$.

(3) The preimages of the edges of $\Delta$ generate the second homology group of $M$. The number of vertices of $\Delta$ is equal to $\operatorname{dim} H_{2}(M)+2$. 
(4) The perimeter of $\Delta($ see $\S 2.4)$ is

$$
\operatorname{perimeter}(\Delta)=\frac{1}{2 \pi} \int_{M} \omega \wedge c_{1}(T M) .
$$

(5) The area of $\Delta$ is

$$
\frac{1}{(2 \pi)^{2}} \int_{M} \frac{1}{2 !} \omega \wedge \omega
$$

Proof. By Lemma 2.2, the preimage of a sufficiently small subinterval $c$ of the edge $d$ is a disk or an annulus with area $2 \pi$ length $(c)$. Part (1) follows.

When $d \neq d^{\prime}$, part (2) follows from Lemma 2.2. When $d=d^{\prime}$, let $d_{-}$ and $d_{+}$be the edges that are adjacent to $d$. Let $u_{-}, u$, and $u_{+}$be the primitive outward normal vectors to the edges $d_{-}, d$, and $d_{+}$. Consider the sub-circle $i: S^{1} \rightarrow \mathbb{T}^{2}$ determined by $u_{-} \in \operatorname{Lie}\left(\mathbb{T}^{2}\right)$. Then $i\left(S^{1}\right)$ acts on the sphere $\Phi^{-1}(d)$ by rotations with speed 1, it rotates the normal to the sphere at the north pole with speed $k$, where $-k$ is the combinatorial self intersection of $d$, and it fixes the normal to the sphere at the south pole. By examining the transition function for a trivializations of the normal bundle over complements of the north and south poles, we see that the Euler number of this normal bundle is $-k$.

By, e.g., Lemma 2.2, a generic component of the moment map is a Morse function with even indices whose critical points are the preimages of the vertices of the polytope. For a critical point of index 2, the descending gradient manifold with respect to an invariant metric is the preimage of an edge of $\Delta$ minus its bottom vertex. Part (3) follows from this using Morse theory.

Let $C_{1}, \ldots, C_{N}$ be the preimages in $M$ of the edges of $\Delta$. Then the homology class $\sum_{i=1}^{N}\left[C_{i}\right] \in H_{2}(M ; \mathbb{R})$ is the Poincaré dual to the first Chern class $c_{1}(T M)$. This follows from the fact that the $C_{i}$ 's generate the second homology group and since

$$
\begin{array}{rlrl}
{\left[C_{i}\right] \cdot \sum_{j=1}^{N}\left[C_{j}\right]} & =\left[C_{i}\right] \cdot\left[C_{i}\right]+2 & & \text { by part }(2) \\
& =c_{1}(T M)\left(\left[C_{i}\right]\right) & & \text { since } C_{i} \text { is an embedded } 2 \text {-sphere in } \\
& \text { a four-manifold. }
\end{array}
$$

This and part (1) imply part (4).

Part (5) follows from Lemma 2.2. It is a special case of the DuistermaatHeckman theorem.

2.11. Let $\Delta$ be a Delzant polytope in $\mathbb{R}^{n}$, let $v$ be a vertex of $\Delta$, and let $\delta>0$ be smaller than the rational lengths of the edges emanating from $v$. The edges of $\Delta$ emanating from $v$ have the form $\left\{v+s \alpha_{j} \mid 0 \leq s \leq \ell_{j}\right\}$ where the vectors $\alpha_{1}, \ldots \alpha_{n}$ generate the lattice $\mathbb{Z}^{n}$ and $\delta<\ell_{j}$ for all $j$. The 


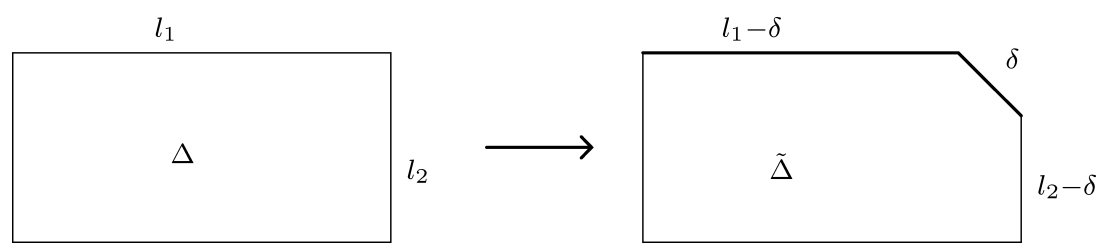

Figure 3. Corner chopping.

corner chopping of size $\delta$ of $\Delta$ at $v$ is the polytope $\tilde{\Delta}$ obtained from $\Delta$ by intersecting with the half-space

$$
\left\{v+s_{1} \alpha_{1}+\cdots+s_{n} \alpha_{n} \mid s_{1}+\cdots+s_{n} \geq \delta\right\} .
$$

See, e.g., the chopping of the top right corner in Figure 3. The resulting polytope $\tilde{\Delta}$ is again a Delzant polytope. The corner chopping operation commutes with $\operatorname{AGL}(n, \mathbb{Z})$-congruence: if $\tilde{\Delta}$ is obtained from $\Delta$ by a corner chopping of size $\delta>0$ at a vertex $v \in \Delta$ then, for any $g \in \operatorname{AGL}(n, \mathbb{Z})$, the polytope $g(\tilde{\Delta})$ is obtained from the polytope $g(\Delta)$ by a corner chopping of size $\delta$ at the vertex $g(v)$.

Remark 2.12. Let a torus $T$ act on a $2 n$-dimensional symplectic manifold $(M, \omega)$. By the equivariant Darboux-Weinstein theorem [38], every $T$-fixed point has a neighborhood $U$ which is equivariantly symplectomorphic to a neighbourhood of the origin in $\mathbb{C}^{n}$ with $T$ acting through a subgroup of $\left(S^{1}\right)^{n}$. The symplectic blow-up of size $r^{2} / 2$ is obtained by removing the open ball $B^{2 n}(r)$ of radius $r$ about the origin and collapsing its boundary along the Hopf fibration $S^{2 n-1} \rightarrow \mathbb{C P}^{n-1}$ (see [31, Section 7.1]). If $M$ is a toric manifold with moment map image $\Delta$, and $\tilde{\Delta}$ can be obtained from $\Delta$ by a corner chopping, then $\tilde{\Delta}$ is the moment map image of a toric manifold obtained from $M$ by an equivariant symplectic blow-up. See [19, Section 3].

The following lemma is an easy consequence of the definitions of corner chopping (§2.11) and of combinatorial self intersection $(\S 2.7)$.

Lemma 2.13. Let $d$ and $d^{\prime}$ be consecutive edges in a Delzant polygon $\Delta$. Let $\tilde{\Delta}$ be obtained from $\Delta$ by a corner chopping at the vertex between $d$ and $d^{\prime}$. Let e be the resulting new edge. Let $\tilde{d}$ and $\tilde{d}^{\prime}$ be the edges of $\tilde{\Delta}$ with the same outward normal vectors as those of $d$ and $d^{\prime}$. Then

(1) the combinatorial self intersection of $e$ is -1 ,

(2) the combinatorial self intersection of $\tilde{d}$ is one less than that of $d$,

(3) the combinatorial self intersection of $\tilde{d}^{\prime}$ is one less than that of $d^{\prime}$,

(4) for any of the other edges of $\tilde{\Delta}$, its combinatorial self intersection is the same as that of the edge of $\Delta$ with the same outward normal vector. 
Remark 2.14. Let $(M, \omega, \Phi)$ be a toric manifold with moment map image $\Delta$. Lemma 2.13 is the combinatorial counterpart of the following geometric facts. Let $\tilde{M}$ be the toric manifold obtained from $M$ by equivariant symplectic blow-up centered at a point $p$. The exceptional divisor in $\tilde{M}$ has self intersection -1; a 2-sphere through $p$ in $M$ with self intersection $\ell$ gives rise to a 2 -sphere in $\tilde{M}$ with self intersection $\ell-1$.

In $\mathbb{R}^{2}$, all Delzant polygons can be obtained by a simple recursive recipe.

Lemma 2.15. (1) Let $\Delta$ be a Delzant polygon with three edges. Then there exists a unique $\lambda>0$ such that $\Delta$ is $\mathrm{AGL}(2, \mathbb{Z})$-congruent to the Delzant triangle $\Delta_{\lambda}$. (See Example 2.5.)

(2) Let $\Delta$ be a Delzant polygon with four or more edges. Let $s$ be the non-negative integer such that the number of edges is $4+s$. Then there exist positive numbers $a \geq b>0$, an integer $0 \leq k \leq 2 a / b$, and positive numbers $\delta_{1}, \ldots, \delta_{s}$, such that $\Delta$ is $\operatorname{AGL}(2, \mathbb{Z})$-congruent to a Delzant polygon that is obtained from the Hirzebruch trapezoid $H_{a, b, k}$ (see Example 2.5) by a sequence of corner choppings of sizes $\delta_{1}, \ldots, \delta_{s}$.

For the proof, see [10, Section 2.5 and Notes to Chapter 2].

Lemma 2.16. Let

$$
\Delta_{0}, \Delta_{1}, \ldots, \Delta_{s}
$$

be a sequence of Delzant polygons such that, for each $i$, the polygon $\Delta_{i}$ is obtained from the polygon $\Delta_{i-1}$ by a corner chopping of size $\delta_{i}$. Then

(1) $\operatorname{area}\left(\Delta_{s}\right)=\operatorname{area}\left(\Delta_{0}\right)-\frac{1}{2} \delta_{1}^{2}-\cdots-\frac{1}{2} \delta_{s}^{2}$.

(2) perimeter $\left(\Delta_{s}\right)=\operatorname{perimeter}\left(\Delta_{0}\right)-\delta_{1}-\cdots-\delta_{s}$.

(3) For each $i, \delta_{i}<2^{s}$ perimeter $\left(\Delta_{s}\right)$.

(4) The length of each edge of each $\Delta_{i}$ is a linear combination, with nonnegative integer coefficients, of the lengths of the edges of $\Delta_{s}$.

Proof. Parts (1) and (2) follow immediately from the definition of corner chopping in $\S 2.11$.

Because a corner chopping of size $\delta_{i}$ can only be performed if there are two consecutive edges in $\Delta_{i-1}$ of lengths greater than $\delta_{i}$,

$$
\operatorname{perimeter}\left(\Delta_{i-1}\right)>2 \delta_{i} \text {. }
$$

Because a corner chopping of size $\delta_{i}$ creates a new edge of size $\delta_{i}$, shortens by $\delta_{i}$ the lengths of two edges, and the other edges do not change,

$$
\operatorname{perimeter}\left(\Delta_{i}\right)=\operatorname{perimeter}\left(\Delta_{i-1}\right)-\delta_{i} .
$$

From these two equalities it follows that

$$
\operatorname{perimeter}\left(\Delta_{i-1}\right)<2 \operatorname{perimeter}\left(\Delta_{i}\right) \text {. }
$$


By induction, we get

$$
\operatorname{perimeter}\left(\Delta_{i}\right)<2^{s-i} \operatorname{perimeter}\left(\Delta_{s}\right) .
$$

Because $\Delta_{i}$ contains an edge of size $\delta_{i}$,

$$
\delta_{i}<\operatorname{perimeter}\left(\Delta_{i}\right)
$$

The last two equalities imply part (3).

Let $d$ be an edge of $\Delta_{i-1}$, let $\tilde{d}$ be the edge of $\Delta_{i}$ with the same outward normal vector, and let $e$ be the edge of $\Delta_{i}$ that was created at the $i$ th corner chopping. If $d$ does not touch the vertex of $\Delta_{i-1}$ at which we chop, then $\operatorname{length}(d)=\operatorname{length}(\tilde{d})$. If $d$ touches the vertex of $\Delta_{i-1}$ at which we chop, then length $(d)=\operatorname{length}(\tilde{d})+\operatorname{length}(e)$. Part (4) of the lemma follows by induction.

2.17. For any Delzant polygon $\Delta$, consider the free Abelian group generated by its edges:

$$
\mathbb{Z}[\text { edges of } \Delta] \text {. }
$$

The combinatorial intersection pairing on this group is the $\mathbb{Z}$-valued bilinear pairing whose restriction to the generators is given by their combinatorial intersection number. The length functional

$$
\mathbb{Z}[\text { edges of } \Delta] \rightarrow \mathbb{R}
$$

is the homomorphism that associates to each basis element its rational length.

Lemma 2.18. If $M$ is a symplectic toric manifold with moment map image $\Delta$, the homomorphism from $\mathbb{Z}[$ edges of $\Delta]$ to $H_{2}(M ; \mathbb{Z})$ that sends a generator $d$ to the homology class of the two-sphere $\Phi^{-1}(d) \subset M$ is onto, it carries the combinatorial intersection pairing on $\mathbb{Z}[$ edges of $\Delta]$ to the ordinary intersection pairing in homology, and it carries the length functional to the functional on homology given by pairing with the symplectic form divided by $2 \pi$.

Proof. The lemma follows from parts (1)-(3) of Lemma 2.10.

If $\Delta_{i+1}$ is obtained from $\Delta_{i}$ by a corner chopping, we consider the injective homomorphism

$$
\mathbb{Z}\left[\text { edges of } \Delta_{i}\right] \hookrightarrow \mathbb{Z}\left[\text { edges of } \Delta_{i+1}\right]
$$

whose restriction to the generators is defined in the following way. If $d$ is an edge of $\Delta_{i}$ that does not touch the corner that was chopped, then $d$ is mapped to the edge of $\Delta_{i+1}$ with the same outward normal vector. If $d$ is an edge of $\Delta_{i}$ that touches the corner that was chopped, then $d$ is mapped to $\tilde{d}+e$ where $e$ is the new edge of $\Delta_{i+1}$, created in the chopping, and $\tilde{d}$ is the edge of $\Delta_{i+1}$ with the same outward normal vector as $d$. 
Lemma 2.19. The homomorphism (2.5) respects the combinatorial intersection pairing and the length homomorphism. Identifying edges of $\Delta_{i}$ with their image under (2.5), we get

$$
\mathbb{Z}\left[\text { edges of } \Delta_{i+1}\right]=\mathbb{Z}\left[\text { edges of } \Delta_{i}\right] \oplus \mathbb{Z}[e] .
$$

The combinatorial intersection pairing of e with an edge of $\Delta_{i}$ is zero; the combinatorial self intersection of $e$ is -1 .

Proof. The definitions of corner chopping (§2.11) and of rational length (§2.4) imply that the homomorphism (2.5) respects the length homomorphism. The assertions about the combinatorial intersection pairing follow from Lemma 2.13.

Remark 2.20. Suppose that $\Delta_{i+1}$ is obtained from $\Delta_{i}$ by a corner chopping. Let $M_{i}$ and $M_{i+1}$ be toric manifolds with moment map images $\Delta_{i}$. and $\Delta_{i+1}$. Then $M_{i+1}$ can be obtained from $M_{i}$ by equivariant symplectic blowup. The homomorphism (2.5) induces the natural inclusion map of $H_{2}\left(M_{i}\right)$ into $H_{2}\left(M_{i+1}\right)$.

Corollary 2.21. Let $(M, \omega)$ be a compact connected symplectic fourmanifold that admits a toric action. Then $b_{2}^{+}(M)=1$.

Recall that $b_{2}^{+}(M)$ is the largest dimension of a subspace of $H_{2}(M ; \mathbb{R})$ on which the intersection form is positive definite.

Proof. Let $\Delta$ be the moment map image for a toric action on $(M, \omega)$. By Part (1) of Proposition 2.3, $\Delta$ is a Delzant polytope. By Lemma 2.18, $b_{2}^{+}(M)$ is equal to the largest dimension of a subspace of $H_{2}(M, \mathbb{R})$ on which the combinatorial intersection pairing is positive definite. By Lemma 2.15, $\Delta$ is either $\operatorname{AGL}(2, \mathbb{Z})$-congruent to a Delzant triangle $\Delta_{\lambda}$, or it is $\operatorname{AGL}(2, \mathbb{Z})$ congruent to a Delzant polytope that is obtained from a Hirzebruch trapezoid $H_{a, b, k}$ by a sequence of corner choppings. By Lemma $2.19, b_{2}^{+}$does not change under corner chopping. Thus, it is enough to show that $b_{2}^{+}=1$ for $\Delta_{\lambda}$ and for $H_{a, b, k}$. This can be checked by direct computation.

\section{Soft proofs of properness and finiteness}

We now state the key lemma used in the proof of our main theorem.

Lemma 3.1. Let $(M, \omega)$ be a compact connected symplectic four-manifold. Then there exists a finite set $\mathcal{D}(M, \omega)$ of positive numbers such that for any polygon $\Delta$, if $\Delta$ is the moment map image of a toric action on $(M, \omega)$, and if $\Delta$ can be obtained from a Delzant polygon $\Delta^{\prime}$ by a sequence of corner choppings of sizes $\delta_{1}, \ldots, \delta_{s}$, then $\delta_{i} \in \mathcal{D}(M, \omega)$ for all $i$.

3.2. The proof of Lemma 3.1 will use the following observation. Let $\Delta$ be a polygon that satisfies the assumptions of Lemma 3.1. By part (3) of Lemma 
2.10 , the number of vertices of $\Delta$ is $\operatorname{dim} H_{2}(M ; \mathbb{R})+2$, so

$$
s<\operatorname{dim} H_{2}(M ; \mathbb{R}) .
$$

By part (3) of Lemma 2.16, by part (4) of Lemma 2.10, and by $(3.1), \delta_{i}$ is smaller than

$$
2^{\operatorname{dim} H_{2}(M ; \mathbb{R})} \cdot \frac{1}{2 \pi} \int_{M} \omega \wedge c_{1}(T M)
$$

for all $i$.

Proof of Lemma 3.1. Suppose that $(M, \omega)$ admits a toric action; otherwise take $\mathcal{D}(M, \omega)$ to be empty. By Corollary $2.21, b_{2}^{+}(M)=1$.

Then, by Lemma 1.2 , for any $K>0$, the set $\left\{E \in H_{2}(M ; \mathbb{R}) \mid E \cdot E=\right.$ -1 and $0 \leq \omega(E) \leq K\}$ is compact. Since $H_{2}(M ; \mathbb{Z})$ is discrete, its intersection with this compact set,

$$
\mathcal{D}_{K}=\left\{E \in H_{2}(M ; \mathbb{Z}) \mid E \cdot E=-1 \text { and } 0 \leq \omega(E) \leq K\right\},
$$

is finite.

Let $\Delta$ be a polygon that satisfies the assumptions of Lemma 3.1. Let $\Delta^{\prime}=\Delta_{0}, \ldots, \Delta_{s}=\Delta$ be a sequence of Delzant polygons such that each $\Delta_{i}$ is obtained from $\Delta_{i-1}$ by a corner chopping of size $\delta_{i}$. Then $\delta_{i}$ is the rational length of the new edge $e_{i}$ of $\Delta_{i}$ created in the corner chopping. By part (1) of Lemma 2.13, the combinatorial self intersection of $e_{i}$ is -1 . By $\S 2.17$ and Lemma 2.18, the composed homomorphism

$$
\mathbb{Z}\left[\text { edges of } \Delta_{i}\right] \rightarrow \mathbb{Z}\left[\text { edges of } \Delta_{s}\right] \rightarrow H_{2}(M ; \mathbb{Z})
$$

sends $e_{i}$ to a class $S \in H_{2}(M ; \mathbb{Z})$ with $\frac{1}{2 \pi} \omega(S)=\delta_{i}$ and $S \cdot S=-1$.

By $\S 3.2$,

$$
\delta_{i}<\frac{1}{2 \pi} K
$$

where

$$
K=2^{\operatorname{dim} H_{2}(M ; \mathbb{R})} \int_{M} \omega \wedge c_{1}(T M) .
$$

Also, $0<\delta_{i}$ for all $i$. Thus, the conclusion of Lemma 3.1 holds when $\mathcal{D}(M, \omega)$ is taken to be the set $\left\{\frac{1}{2 \pi} \omega(E) \mid E \in \mathcal{D}_{K}\right\}$.

Remark 3.3. If there exists a positive number $h$ such that $\int_{A} \omega$ is an integer multiple of $h$ for all $A \in H_{2}(M ; \mathbb{Z})$, there is an even shorter proof of Lemma 3.1: after replacing $\omega$ by $\frac{2 \pi}{h} \omega$ (which does not affect the symplectomorphism group) we may assume that $\frac{1}{2 \pi} \int_{A} \omega \in \mathbb{Z}$ for all $A \in H_{2}(M ; \mathbb{Z})$. By part (1) of Lemma 2.10, the lengths of all the edges of $\Delta$ are integers. By part (4) of Lemma 2.16, this implies that all the $\delta_{i}$ 's are integers. Thus, the conclusion of Lemma 3.1 holds when $\mathcal{D}(M, \omega)$ is taken to be the set of positive integers that are smaller than (3.2). 
We now give an elementary algebraic proof of the properness lemma:

Proof of Lemma 1.2. Let $(M, \omega)$ be a symplectic 4-manifold with $b_{2}^{+}=1$. Let $A \in H_{2}(M ; \mathbb{R})$ be the Poincaré dual to $[\omega]$. Notice that $A \cdot A=\omega(A)=$ $\int_{M} \omega \wedge \omega$ is positive, and that $A \cdot C=\omega(C)$ for all $C \in H_{2}(M ; \mathbb{R})$. Without loss of generality, $A \cdot A=1$. Complete $A$ to an orthogonal basis $\mathcal{B}=$ $\left\{A, B_{1}, \ldots, B_{k}\right\}$ of $H_{2}(M ; \mathbb{R})$ with the intersection pairing such that $B_{j} \cdot B_{j}=$ -1 for all $j$. The period map $E \mapsto \omega(E)$ takes $E=x_{0} A+\sum_{j=1}^{k} x_{j} B_{j}$ to the coefficient $x_{0}$. The restriction of this map to the hyperboloid that consists of the classes in $H_{2}(M ; \mathbb{R})$ with self intersection -1 is proper: if $x_{0}^{2}-\sum_{i=1}^{k} x_{i}^{2}=-1$ and $x_{0}$ is bounded, then so are $x_{1}, \ldots, x_{k}$.

Remark 3.4. For a manifold obtained from $\mathbb{C P}^{2}$ by eight or fewer blow-ups, the Poincaré dual $C_{1}$ to the first Chern class $c_{1}(T M)$ is of positive self intersection. This implies that the set of homology classes $E \in H_{2}(M ; \mathbb{R})$ that satisfy $E \cdot E=-1$ and $c_{1}(T M)(E)=1$ is compact. (The argument is similar to the proof of Lemma 1.2, with $A$ replaced by $C_{1}$.) Hence the intersection of this set with the discrete $H_{2}(M ; \mathbb{Z})$ is finite. In particular, there exist only finitely many $E \in H_{2}(M ; \mathbb{Z})$ of self intersection -1 that can be represented by a symplectically embedded sphere. See $[\mathbf{9}]$ or $[\mathbf{2 5}$, ch. IV,$\S 26]$.

We can now deduce our main theorem.

Proof of Theorem 1.1. Fix a compact connected symplectic four-manifold $(M, \omega)$.

Let $\Delta$ be the moment map image for a toric action on $(M, \omega)$.

By part (1) of Proposition 2.3, $\Delta$ is a Delzant polygon. By parts (4) and (5) of Lemma 2.10,

$$
\operatorname{perimeter}(\Delta)=\frac{1}{2 \pi} \int_{M} \omega \wedge c_{1}(T M)
$$

and

$$
\operatorname{area}(\Delta)=\frac{1}{(2 \pi)^{2}} \int_{M} \frac{1}{2 !} \omega \wedge \omega
$$

First, suppose that $\operatorname{dim} H_{2}(M ; \mathbb{R})=1$. By part (3) of Lemma 2.10, $\Delta$ has three edges. By part (1) of Lemma $2.15, \Delta$ is $\operatorname{AGL}(2, \mathbb{Z})$-congruent to a standard Delzant triangle, $\Delta_{\lambda}$. So perimeter $(\Delta)=3 \lambda$ and area $(\Delta)=$ $\frac{1}{2} \lambda^{2}$. By this and (3.3) or (3.4), $\lambda$ is uniquely determined by the symplectic manifold $(M, \omega)$. By part (2) of Proposition 2.3, up to equivalence, there exists only one toric action on $(M, \omega)$.

Now suppose that $\operatorname{dim} H_{2}(M ; \mathbb{R}) \geq 2$. By part (3) of Lemma $2.10, \Delta$ has $(4+s)$ vertices, where $s$ is the non-negative integer

$$
s=\operatorname{dim} H_{2}(M ; \mathbb{R})-2 .
$$


By part (2) of Lemma 2.15, after possibly transforming $\Delta$ by an element of $\operatorname{AGL}(2, \mathbb{Z})$, there exist positive numbers $a \geq b>0$, an integer $0 \leq k<2 a / b$, and (if $s>0$ ) positive numbers $\delta_{1}, \ldots, \delta_{s}>0$ such that $\Delta$ can be obtained from the Hirzebruch trapezoid $H_{a, b, k}$ by a sequence of corner choppings of sizes $\delta_{1}, \ldots, \delta_{s}$. By part (2) of Lemma 2.16,

$$
\operatorname{perimeter}(\Delta)=\operatorname{perimeter}\left(H_{a, b, k}\right)-\sum \delta_{i}=2(a+b)-\sum \delta_{i} .
$$

By part (1) of Lemma 2.16,

$$
\operatorname{area}(\Delta)=\operatorname{area}\left(H_{a, b, k}\right)-\frac{1}{2} \sum \delta_{i}^{2}=a b-\frac{1}{2} \sum \delta_{i}^{2} .
$$

By this, (3.3), and (3.4), the sum and product, $a+b$ and $a b$, and hence also the numbers $a$ and $b$ themselves, are determined by the symplectic manifold $(M, \omega)$ and the values of $\delta_{1}, \ldots, \delta_{s}$. Because, by Lemma 3.1 and by $(3.5)$, the number of possible tuples $\left(\delta_{1}, \ldots, \delta_{s}\right)$ that can arise in this way is finite, there are only finitely many possibilities for the values of $a$ and $b$. Because $k$ is an integer and it satisfies

$$
0 \leq k<\frac{2 a}{b}
$$

there are only finitely many possible values for $k$.

We have shown that, after transforming $\Delta$ by an element of $\operatorname{AGL}(2, \mathbb{Z})$, there exists a tuple $\left(a, b, k ; \delta_{1}, \ldots, \delta_{s}\right)$ such that $\Delta$ can be obtained from $H_{a, b, k}$ by a sequence of corner choppings of sizes $\delta_{1}, \ldots, \delta_{s}$, and that the number of tuples $\left(a, b, k ; \delta_{1}, \ldots, \delta_{s}\right)$ that can arise in this way is finite. For any given polygon, the number of possible corner choppings of a given size is at most the number of vertices, and hence it is finite. It follows that, modulo $\operatorname{AGL}(2, \mathbb{Z})$-congruence, the number of polygons $\Delta$ that can arise as moment map images of toric actions on $(M, \omega)$ is finite.

By part (2) of Proposition 2.3, the number of toric actions on $(M, \omega)$ is finite.

As explained in the introduction, Theorem 1.1 implies that for a fourdimensional compact connected symplectic manifold $(M, \omega)$ with $H^{1}(M ; \mathbb{R})$ $=\{0\}$, the group $\operatorname{Sympl}(M, \omega)$ can contain only finitely many non-conjugate two-dimensional maximal tori. We conclude this section with examples of symplectomorphism groups in which not all maximal tori are twodimensional. In the first symplectomorphism group of Example 3.5, one maximal torus is two-dimensional and another maximal torus is one-dimensional. The second symplectomorphism group contains onedimensional maximal tori and does not contain any two-dimensional tori. The third symplectomorphism group does not contain any tori. The symplectic manifolds in these examples are obtained from $\mathbb{C P}^{2}$ by symplectic blow-ups of equal sizes. 


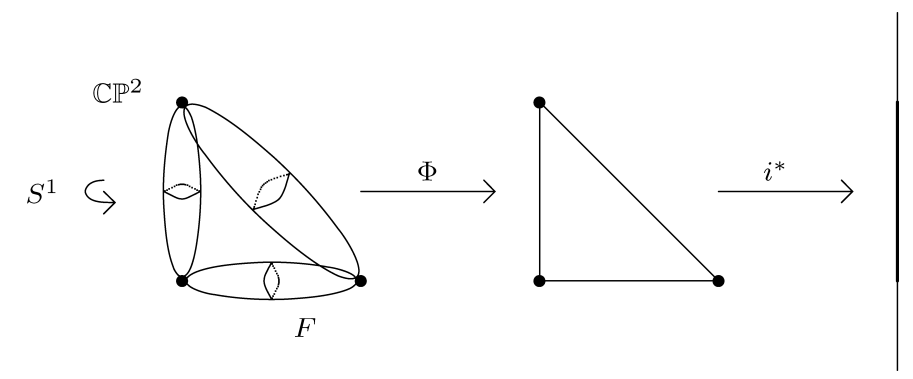

Figure 4. Moment maps for circle and torus actions on $\mathbb{C P}^{2}$.

Example 3.5. Take the $\mathbb{T}^{2}$-action on $\mathbb{C P}^{2}$ given by $(a, b) \cdot\left[z_{0}, z_{1}, z_{2}\right]=$ $\left[z_{0}, a z_{1}, b z_{2}\right]$. There are exactly three fixed points. If we restrict to the $S^{1}$ action given by $a \cdot\left[z_{0}, z_{1}, z_{2}\right]=\left[z_{0}, z_{1}, a z_{2}\right]$, the $S^{1}$-fixed point set consists of the point $[0,0,1]$ and the component $F=\left\{\left[z_{0}, z_{1}, 0\right]\right\} \cong \mathbb{C P}^{1}$. The moment map for this $S^{1}$ action attains its minimum along $F$. See Figure ?? (where $\Phi: \mathbb{C P}^{2} \rightarrow \mathbb{R}^{2}$ is the $\mathbb{T}^{2}$ moment map, $i: S^{1} \rightarrow \mathbb{T}^{2}$ is the inclusion map, and $i^{*} \circ \Phi: \mathbb{C P}^{2} \rightarrow \mathbb{R}$ is the $S^{1}$ moment map).

Let $T=S^{1}$ or $T=\mathbb{T}^{2}$. For any finite set of $T$-fixed points, we can choose $T$-equivariant Darboux charts that are centered at these points and are disjoint from each other; if $\epsilon>0$ is sufficiently small, we can use these Darboux charts to perform $T$-equivariant symplectic blow-ups of size $\epsilon$ centered at the chosen fixed points.

(1) Choose a sufficiently small positive number $\epsilon$ and perform three $S^{1}$ equivariant symplectic blow-ups of size $\epsilon$ that are centered at points of $F$. Each such a blow-up creates an isolated fixed point whose moment map value is equal to that of $F$ plus $\epsilon$. If a Hamiltonian $S^{1}$ action on a symplectic four-manifold has three or more fixed points with the same moment map value, the action does not extend to a Hamiltonian $\mathbb{T}^{2}$-action; see $[\mathbf{4}]$ or $[\mathbf{1 7}$, Proposition 5.21]. Thus, this $S^{1}$-action is a maximal torus. It is a one-dimensional maximal torus in the symplectomorphism group of a symplectic manifold that is obtained from $\mathbb{C P}^{2}$ by three symplectic blow-ups of size $\epsilon$.

On the other hand, perform three $\mathbb{T}^{2}$-equivariant symplectic blowups of size $\epsilon$, centered at the three $\mathbb{T}^{2}$-fixed points. This yields a two-dimensional maximal torus in the symplectomorphism group of a symplectic manifold that is obtained from $\mathbb{C P}^{2}$ by three symplectic blow-ups of size $\epsilon$.

By [29], (also see [5],) any two symplectic manifolds that are obtained from $\mathbb{C P}^{2}$ by symplectic blow-ups of the same sizes are symplectomorphic. Therefore, the above constructions give maximal tori in the symplectomorphism group of the same symplectic manifold; 
one of these maximal tori is one-dimensional and the other is twodimensional.

(2) If $\epsilon$ is sufficiently small, we can perform four $S^{1}$-equivariant symplectic blow-ups centered at points of $F$. Alternatively, we can perform three $S^{1}$-equivariant symplectic blow-ups centered at points of $F$ and one that is centered at the isolated fixed point $[0,0,1]$. These two constructions yield two circle actions that are not equivalent (e.g., the lists of moment map values at their fixed points are different). If $1 / \epsilon$ is an integer, a manifold that is obtained from $\mathbb{C P}^{2}$ by four or more symplectic blow-ups of size $\epsilon$ does not admit a toric action; see [19, Theorem 4.1(1)]. Thus, the symplectomorphism group of such a symplectic manifold, when $\epsilon$ is sufficiently small and $1 / \epsilon$ is an integer, contains at least two non-conjugate one-dimensional maximal tori, and it does not contain any two-dimensional maximal tori.

(3) If $1 / \epsilon$ is an integer and $(k-1) \epsilon \geq 1$, then a symplectic manifold that is obtained from $\mathbb{C P}^{2}$ by $k$ symplectic blow-ups of size $\epsilon$ does not admit any circle action; see [19, Theorem $4.1(2)]$. It is possible to perform four symplectic blow-ups of $\mathbb{C P}^{2}$ of size $\epsilon=1 / 3$ (see, e.g., [37]). Thus, the resulting symplectic manifold does not admit any circle action.

\section{A. Appendix. $J$-holomorphic spheres in symplectic four-manifolds}

In this appendix, we introduce the non-experts to a beautiful work of McDuff that establishes properness for a general compact symplectic four-manifold.

We then apply this result, together with a lemma on the existence of certain symplectically embedded 2-spheres, to give another proof of Lemma 3.1.

Lemma A.1. Let $(M, \omega)$ be a closed symplectic four-manifold. Let $\mathcal{E}$ denote the set of homology classes $E \in H_{2}(M ; \mathbb{Z})$ that are represented by symplectic exceptional spheres. Then the map $E \mapsto \omega(E)$ from $\mathcal{E}$ to $\mathbb{R}$ is proper.

When $b_{2}^{+}(M)=1$, Lemma A.1 follows from Lemma 1.2.

A symplectic exceptional sphere is a symplectically embedded sphere with self intersection -1 .

Lemma A.1 is proved in [26, Lemma 3.1; 29, $\S 3]$ using Gromov's theory of J-holomorphic curves. We recall relevant parts of this theory.

Let $(M, \omega)$ be a compact symplectic manifold. Let $\mathcal{J}=\mathcal{J}(M, \omega)$ be the space of almost complex structures on $M$ that are tamed by $\omega$; see $\S 2.9$. Given $J \in \mathcal{J}$, a parametrized $J$-holomorphic sphere is a map $u: \mathbb{C P}^{1} \rightarrow M$ such that $d u: T \mathbb{C P}^{1} \rightarrow T M$ satisfies the Cauchy-Riemann equation $d u \circ i=J \circ d u$. Such a $u$ represents a homology class in $H_{2}(M ; \mathbb{Z})$ that we denote $[u]$. The symplectic area $\omega([u]):=\int_{\mathbb{C P}^{1}} u^{*} \omega$ only depends on the homology class $[u] \in H_{2}(M ; \mathbb{Z})$. The pull-back $u^{*} \omega$ is a positively oriented area form at each point where $d u \neq 0$; it follows that the class 
$[u]$ is non-zero if and only if $u$ is not constant, if and only if $\omega([u])>0$. A $J$-holomorphic sphere is called simple if it cannot be factored through a branched covering of $\mathbb{C P}^{1}$. One similarly defines a holomorphic curve in $(M, J)$ whose domain is a Riemann surface other than $\mathbb{C P}^{1}$. Gromov, in [13], introduced a notion of "weak convergence" of a sequence of holomorphic curves. This notion is preserved under reparametrization of the curve, and it implies convergence in homology. Gromov's compactness theorem guarantees that, given a converging sequence of almost complex structures, a corresponding sequence of holomorphic curves with bounded symplectic area has a weakly converging subsequence.

The limit under weak convergence might not be a curve; it might be a "cusp curve", which is a connected union of holomorphic curves. In this paper, we do not need the precise definitions of cusp curve and of weak convergence. Readers who are not experienced with these notions may find this example helpful:

Example A.2. Identify $\mathbb{C P}^{1}$ with $\mathbb{C} \sqcup\{\infty\}$. For each $0<\lambda<\infty$, consider the holomorphic curve in $M=\mathbb{C P}^{1} \times \mathbb{C P}^{1}=(\mathbb{C} \sqcup\{\infty\}) \times(\mathbb{C} \sqcup\{\infty\})$ given by $\{(w, z) \mid w=\lambda z\}$. This curve can be parametrized by $z \mapsto(\lambda z, z)$ or, alternatively, by $w \mapsto\left(w, \lambda^{-1} w\right)$. As $\lambda \rightarrow 0$, these curves weakly converge to the cusp curve $\{0\} \times \mathbb{C P}^{1} \cup \mathbb{C P}^{1} \times\{\infty\}$.

Lemma A.3. Let $\left\{J_{n}\right\} \subset \mathcal{J}$ be a sequence of almost complex structures that converges in the $C^{\infty}$ topology to an almost complex structure $J_{\infty} \in \mathcal{J}$. For each $n$, let $f_{n}: \mathbb{C P}^{1} \rightarrow M$ be a parametrized $J_{n}$-holomorphic sphere. Suppose that the set of areas $\omega\left(\left[f_{n}\right]\right)$ is bounded. Then one of the following two possibilities occurs.

(1) There exists a $J_{\infty}$-holomorphic sphere $u: \mathbb{C P}^{1} \rightarrow M$ and elements $A_{n} \in \operatorname{PSL}(2, \mathbb{C})$ such that a subsequence of the $f_{n} \circ A_{n}$ 's converges to $u$ in the $C^{\infty}$ topology. In particular, there exist infinitely many $n$ 's for which $\left[f_{n}\right]=[u]$.

(2) There exist two or more non-constant simple $J_{\infty}$-holomorphic spheres $u_{\ell}: \mathbb{C P}^{1} \rightarrow M$ and positive integers $m_{\ell}$, for $\ell=1, \ldots, L$, and infinitely many $n$ 's for which

$$
\left[f_{n}\right]=\sum_{\ell=1}^{L} m_{\ell}\left[u_{\ell}\right] \quad \text { in } H_{2}(M ; \mathbb{Z}) .
$$

Proof. If there exists a subsequence of the $f_{n}$ 's that, after reparametrization, converges in the $C^{\infty}$ topology to a map $u: \mathbb{C P}^{1} \rightarrow M$, then the map $u$ is $J_{\infty}$-holomorphic, and the first of the two possibilities occurs.

Otherwise, by Gromov's compactness theorem, there exists a subsequence that weakly converges to a cusp curve. See $[\mathbf{3 6}, \S 5]$. This cusp curve has at least two components; its components are non-constant $J_{\infty}$-holomorphic 
maps $v_{\ell}: \mathbb{C P}^{1} \rightarrow M$; weak convergence implies convergence in homology, so that $\left[f_{n}\right]=\sum_{\ell}\left[v_{\ell}\right]$ for all but a finite number of $f_{n}$ 's in the subsequence.

If $v_{\ell}$ is not simple then there exists a simple non-constant $J_{\infty}$-holomorphic $u_{\ell}: \mathbb{C P}^{1} \rightarrow M$ and a positive integer $m_{\ell}$ such that $\left[v_{\ell}\right]=m_{\ell}\left[u_{\ell}\right]$.

Corollary A.4. Let $(M, \omega)$ be a closed symplectic manifold. Let $K$ be a positive real number. For each $J \in \mathcal{J}(M, \omega)$, there exist only finitely many homology classes $A \in H_{2}(M ; \mathbb{Z})$ such that $\omega(A) \leq K$ and such that the class $A$ is represented by a $J$-holomorphic sphere.

Proof. Otherwise, there exists a sequence of $J$-holomorphic spheres $f_{n}: \mathbb{C P}^{1}$ $\rightarrow M$ such that $\omega\left(\left[f_{n}\right]\right) \leq K$ for each $n$ and such that the classes $\left[f_{n}\right]$ are all different. This contradicts Lemma A.3.

Fix a compact symplectic manifold $(M, \omega)$. For any class $A \in H_{2}(M ; \mathbb{Z})$, consider the universal moduli space of simple parametrized holomorphic spheres in the class $A$,

$$
\begin{gathered}
\mathcal{M}(A, \mathcal{J})=\left\{(u, J) \mid J \in \mathcal{J}, u: \mathbb{C P}^{1} \rightarrow M\right. \text { is simple } \\
J \text {-holomorphic, and }[u]=A\},
\end{gathered}
$$

and the projection map

$$
p_{A}: \mathcal{M}(A, \mathcal{J}) \rightarrow \mathcal{J}
$$

For any positive number $K$, let

$$
\mathcal{N}_{K}=\left\{A \in H_{2}(M ; \mathbb{Z}) \mid A \neq 0, c_{1}(T M)(A) \leq 0, \text { and } \omega(A)<K\right\} .
$$

The importance of this set lies in the fact that if a homology class $E$ with $\omega(E) \leq K$ and $c_{1}(T M)(E) \leq 1$ is represented by a $J$-holomorphic cuspcurve with two or more components then at least one of these components must lie in a homology class in $\mathcal{N}_{K}$ :

Lemma A.5. Let $J \in \mathcal{J}$. Let $u_{\ell}: \mathbb{C P}^{1} \rightarrow M, \ell=1, \ldots, L$ be two or more non-constant $J$-holomorphic spheres, and let $m_{1}, \ldots, m_{L}$ be positive integers. Consider the homology class $E=\sum m_{\ell}\left[u_{\ell}\right]$ in $H_{2}(M ; \mathbb{Z})$. Suppose that $c_{1}(T M)(E) \leq 1$. Let $K=\omega(E)$. Then there exists $1 \leq \ell \leq L$ such that $\left[u_{\ell}\right] \in \mathcal{N}_{K}$.

Proof. By assumption,

$$
\sum_{\ell=1}^{L} m_{\ell} \omega\left(\left[u_{\ell}\right]\right)=K .
$$

Because the left-hand side consists of two or more positive summands, each summand is smaller than the sum $K$ :

$$
\omega\left(\left[u_{\ell}\right]\right)<K \text { for all } \ell \text {. }
$$


By assumption,

$$
\sum_{\ell=1}^{L} m_{\ell} c_{1}(T M)\left(\left[u_{\ell}\right]\right) \leq 1 .
$$

Because this is a positive combination of two or more integers and the sum is no greater than one, the integers cannot all be greater than or equal to one, so there must exist at least one $\ell$ such that

$$
c_{1}(T M)\left(\left[u_{\ell}\right]\right) \leq 0 .
$$

By this and (A.2), the class $\left[u_{\ell}\right]$ is in $\mathcal{N}_{K}$.

The automorphism group $\operatorname{PSL}(2, \mathbb{C})$ of $\mathbb{C P}^{1}$ acts on $\mathcal{M}(A, \mathcal{J})$ by reparametrizations. The quotient $\mathcal{M}(A, \mathcal{J}) / \operatorname{PSL}(2, \mathbb{C})$ is the space of unparametrized $J$-holomorphic spheres representing $A \in H_{2}(M ; \mathbb{Z})$. Let

$$
U_{K}=\mathcal{J} \backslash \bigcup_{A \in \mathcal{N}_{K}} \text { image } p_{A} .
$$

Lemma A.6. Let $E \in H_{2}(M ; \mathbb{Z})$ be a homology class such that $c_{1}$ $(T M)(E) \leq 1$. Let $K=\omega(E)$. Then the map

$$
p_{E}^{-1}\left(U_{K}\right) / \operatorname{PSL}(2, \mathbb{C}) \rightarrow U_{K}
$$

that is induced from the projection map $p_{E}: \mathcal{M}(E, \mathcal{J}) \rightarrow \mathcal{J}$ is proper.

Proof. The lemma follows from Gromov's compactness in the following way. Let $D \subset U_{K}$ be a compact subset. We need to show that $p_{E}^{-1}(D) / \operatorname{PSL}(2, \mathbb{C})$ is compact. Because $\mathcal{M}(E, \mathcal{J})$ is Hausdorff and first countable, it is enough to show that for every sequence $\left\{\left(f_{n}, J_{n}\right)\right\}$ in $p_{E}^{-1}(D)$ there exists a subsequence that, after reparametrization, has a limit in $p_{E}^{-1}(D)$ in the $C^{\infty}$ topology.

Take such a sequence, $\left\{\left(f_{n}, J_{n}\right)\right\}$. Because $J_{n} \in D$ and $D$ is compact and contained in $U_{K}$, after passing to a subsequence we may assume that $\left\{J_{n}\right\}$ converges to $J_{\infty} \in U_{K}$.

Suppose that there exists a subsequence that, after reparametrization, converges to some $u: \mathbb{C P}^{1} \rightarrow M$ in the $C^{\infty}$ topology. Because each $f_{n}$ is a $J_{n}$-holomorphic sphere in the class $E$, the limit $u$ must also be in the class $E$ and it must be $J_{\infty}$-holomorphic.

We need to show that $u$ is simple. If not, there is a simple $J_{\infty}$-holomorphic curve $v$ and an integer $m \geq 2$ such that $[u]=m[v]$. Because $v$ is simple and $J_{\infty}$-holomorphic, the pair $\left(v, J_{\infty}\right)$ is in the moduli space $\mathcal{M}(A, \mathcal{J})$ for $A=$ $[v]$, so $J_{\infty} \in$ image $p_{A}$. The inequalities $c_{1}(T M)([u]) \leq 1$ and $\omega([u])=K$ imply that $c_{1}(T M)([v]) \leq 0$ and $\omega([v])<K$, so that $A=[v]$ is in $\mathcal{N}_{K}$. So $J_{\infty} \in$ image $p_{A}$ cannot be in $U_{K}$. Therefore, $u$ is simple.

The pair $\left(u, J_{\infty}\right)$ is then in the moduli space $\mathcal{M}(E, \mathcal{J})$, and since $J_{\infty} \in D$, this pair is in $p_{E}^{-1}(D)$. 
Now suppose that there does not exist such a subsequence, and we would like to arrive at a contradiction. By Lemma A.3, there exist two or more nonconstant simple $J_{\infty}$-holomorphic spheres $u_{\ell}: \mathbb{C P}^{1} \rightarrow M$ and positive integers $m_{\ell}$ such that $\sum m_{\ell}\left[u_{\ell}\right]=E$. Because $\omega(E)=K$ and $c_{1}(T M)(E) \leq 1$, by Lemma A.5 at least one of the $u_{\ell}$ 's represents a homology class in $\mathcal{N}_{K}$. This contradicts the fact that the $u_{\ell}$ 's are $J_{\infty}$-holomorphic for $J_{\infty} \in U_{K}$ and the definition of $U_{K}$.

Corollary A.7. Let $E$ be a homology class such that $c_{1}(T M)(E)=1$ and $\omega(E) \leq K$. Then image $p_{E} \cap U_{K}$ is closed in $U_{K}$.

Lemma A.8. The subset $U_{K} \subset \mathcal{J}$ is open.

Proof. Let $\left\{J_{n}\right\}$ be a sequence of almost complex structures that converges in the $C^{\infty}$ topology to an almost complex structure $J_{\infty}$. We need to show that if each $J_{n}$ lies in the union

$$
\bigcup_{A \in \mathcal{N}_{K}} \text { image } p_{A}
$$

then so does $J_{\infty}$. For each $n$, let $f_{n}: \mathbb{C P}^{1} \rightarrow M$ be a $J_{n}$-holomorphic sphere such that

$$
\omega\left(\left[f_{n}\right]\right)<K \quad \text { and } \quad c_{1}(T M)\left(\left[f_{n}\right]\right) \leq 0 .
$$

By Lemma A.3, there exist one or more non-constant simple $J_{\infty}$-holomorphic spheres $u_{\ell}: \mathbb{C P}^{1} \rightarrow M$ and positive integers $m_{\ell}$ such that

$$
\sum_{\ell=1}^{L} m_{\ell}\left[u_{\ell}\right]=\left[f_{n}\right]
$$

for infinitely many $n$ 's. By Lemma A.5 there exists at least one $u_{\ell}$ whose homology class $\left[u_{\ell}\right]$ is in $\mathcal{N}_{K}$. Because $u_{\ell}$ is $J_{\infty}$-holomorphic and $u_{\ell}$ is simple, $J_{\infty} \in$ image $p_{\left[u_{\ell}\right]}$.

We have the following consequences of the Sard-Smale theorem, the infinite-dimensional inverse mapping theorem, and the ellipticity of the Cauchy-Riemann equations. See the arXiv version of this paper, [20], for a derivation of these consequences, using the results of [32, Chapter 3].

Lemma A.9. (a) The universal moduli space $\mathcal{M}(A, \mathcal{J})$ and the space $\mathcal{J}$ of compatible almost complex structures are Fréchet manifolds, and the projection map

$$
p_{A}: \mathcal{M}(A, \mathcal{J}) \rightarrow \mathcal{J}
$$

is a Fredholm map of index

$$
\operatorname{dim} \operatorname{ker} d p_{A}-\operatorname{dim} \text { coker } d p_{A}=2 c_{1}(T M)(A)+2 n,
$$

where $2 n$ is the dimension of $M$. 
(b) If $(u, J)$ is a regular value for $p_{A}$, then for any neighborhood $U$ of $(u, J)$ in $\mathcal{M}(A, \mathcal{J})$, its image, $p_{A}(U)$, contains a neighborhood of $J$ in $\mathcal{J}$.

(c) The set of $J$ 's that are regular for the map $p_{A}: \mathcal{M}(A, \mathcal{J}) \rightarrow \mathcal{J}$ for all $A \in H_{2}(M ; \mathbb{Z})$ is dense in $\mathcal{J}$.

(d) Let $\mathcal{A}$ be a subset of $H_{2}(M ; \mathbb{Z})$. Let $\left\{J_{t}\right\}_{t \in[0,1]}$ be a $C^{1}$ simple path in $\mathcal{J}$ whose endpoints are regular values for $p_{A}$ for all $A \in \mathcal{A}$. Then there exists a $C^{1}$ perturbation $\left\{\tilde{J}_{t}\right\}$ of $J_{t}$ with the same endpoints which is transversal to $p_{A}$ for all $A \in \mathcal{A}$.

Lemma A.10. ( $C_{1}$ lemma). Let $(M, \omega)$ be a compact symplectic fourmanifold. The subset $U_{K} \subset \mathcal{J}$ is dense and path connected.

Proof. Let $A \in \mathcal{N}_{K}$. Since $A \neq 0$, the $\operatorname{PSL}(2, \mathbb{C})$-orbits in $\mathcal{M}(A, J)$ are sixdimensional submanifolds of the level sets $p_{A}^{-1}(J)$, and so $\left.\operatorname{dim} \operatorname{ker} d p_{A}\right|_{(u, J)} \geq$ 6 for all $(u, J) \in \mathcal{M}(A, \mathcal{J})$. Since $c_{1}(T M)(A) \leq 0$ then, by (A.6), $\operatorname{dim}$ coker $d p_{A} \geq 2$ for all $(u, J) \in \mathcal{M}(A, \mathcal{J})$.

In particular, $d p_{A}$ is never onto. So a point in $\mathcal{J}$ is a regular value for $p_{A}$ if and only if it is not in the image of $p_{A}$. The fact that $U_{K}$ is dense then follows from part (c) of Lemma A.9.

Let $J_{0}$ and $J_{1}$ be two points in $U_{K}$. Let $\left\{J_{t}\right\}_{t \in[0,1]}$ be any simple path in $\mathcal{J}$ connecting $J_{0}$ to $J_{1}$. By part (d) of Lemma A.9, there exists a path $\left\{\tilde{J}_{t}\right\}_{t \in[0,1]}$ connecting $J_{0}$ to $J_{1}$ that is transversal to $p_{A}$ for all $A \in \mathcal{N}_{K}$. Because $\operatorname{dim}$ coker $d p_{A} \geq 2$ for all $A \in \mathcal{N}_{K}$, transversality means that the path is disjoint from image $p_{A}$. So the path lies in $U_{K}$.

Lemma A.11. Let $(M, \omega)$ be a compact symplectic four-manifold. Let $A \in H_{2}(M ; \mathbb{Z})$ be a homology class that is represented by an embedded symplectic sphere $C$. Then for any $J \in \mathcal{J}$ and any simple $J$-holomorphic $u: \mathbb{C P}^{1} \rightarrow M$ in the class $A$, the map $u$ is an embedding.

Proof. Let $u_{0}: \mathbb{C P}^{1} \rightarrow M$ be a symplectic embedding whose image is $C$. Construct an almost complex structure $J_{0} \in \mathcal{J}$ for which $u_{0}$ is a $J_{0}$-holomorphic sphere. (Define $\left.J_{0}\right|_{T C}$ such that $u_{0}$ is holomorphic; extend it to a compatible fiberwise complex structure on the symplectic vector bundle $\left.T M\right|_{C}$; then extend it to a compatible almost complex structure on $M$. See [31, Section 2.6].)

By the adjunction inequality, for any $(u, J) \in \mathcal{M}(A, \mathcal{J})$,

$$
A \cdot A-c_{1}(T M)(A)+2 \geq 0,
$$

with equality if and only if $u$ is an embedding. See [28, Theorem 2.2.1] or [32, Corollary E.1.7]. Applying this to $\left(u_{0}, J_{0}\right)$, we get that the homology class $A$ satisfies $A \cdot A-c_{1}(T M)(A)+2=0$. Applying the adjunction inequality to any other $(u, J) \in \mathcal{M}(A, \mathcal{J})$, we get that $u$ is an embedding. 
Lemma A.12. Let $(M, \omega)$ be a compact symplectic four-manifold. Let $E \in H_{2}(M ; \mathbb{Z})$ be a homology class that can be represented by an embedded symplectic sphere and such that $c_{1}(T M)(E)=1$. Then

(1) The projection map $p_{E}: \mathcal{M}(E, \mathcal{J}) \rightarrow \mathcal{J}$ is open.

(2) Let $K \geq \omega(E)$. Then, for any $J \in U_{K}$, the class $E$ is represented by an embedded $J$-holomorphic sphere.

Proof. Let $(u, J) \in \mathcal{M}(E, \mathcal{J})$. By Lemma A.11, the map $u: \mathbb{C P}^{1} \rightarrow M$ is an embedding. By the Hofer-Lizan-Sikorav regularity criterion [16], if $u$ is an immersed $J$-holomorphic sphere and $c_{1}([u]) \geq 1$, then $(u, J)$ is a regular point for the map $p_{E}: \mathcal{M}(E, \mathcal{J}) \rightarrow \mathcal{J}$. By part (b) of Lemma A.9 it follows that the map $p_{E}$ is open.

Therefore, image $p_{E}$ is an open subset of $\mathcal{J}$. Because, by Lemmas A.8 and A.10, $U_{K}$ is open and dense in $\mathcal{J}$, the set image $p_{E} \cap U_{K}$ is a non-empty open subset of $U_{K}$. Because, by Lemma A.7, image $p_{E} \cap U_{K}$ is also closed in $U_{K}$, and because, by Lemma A.10, the set $U_{K}$ is connected, we conclude that image $p_{E} \cap U_{K}=U_{K}$. So for every $J \in U_{K}$ there exists a simple $J$-holomorphic sphere $u: \mathbb{C P}^{1} \rightarrow M$ in the class $E$. By Lemma A.11, $u$ is an embedding.

We can now complete the proof of the general properness lemma.

Proof of Lemma A.1. Let $K$ be a positive real number. Suppose that $E_{1}, E_{2}, \ldots$ are infinitely many distinct classes in $H_{2}(M ; \mathbb{Z})$ that satisfy the following conditions.

(1) The class $E$ can be represented by an embedded symplectic sphere in $M$;

(2) The self intersection $E \cdot E$ is -1 ;

(3) The symplectic area $\omega(E)$ is not larger than $K$.

For each $i$, because $E_{i} \cdot E_{i}=-1$ and $E_{i}$ is represented by an embedded symplectic sphere, we have $c_{1}(T M)\left(E_{i}\right)=1$. Pick any $J \in U_{K}$. By part (2) of Lemma A.12, for each $i$, there exists an embedded $J$-holomorphic sphere in the class $E_{i}$. By Corollary A.4, the $E_{i}$ 's cannot all be distinct.

To deduce Lemma 3.1, we will also need the following result, about the existence of certain symplectic exceptional spheres.

Lemma A.13. Let $(M, \omega)$ be a compact symplectic four-manifold. Suppose that $(M, \omega)$ is obtained by a sequence of symplectic blow-ups from a symplectic manifold $\left(M_{0}, \omega_{0}\right)$. Let $C_{0}$ be a symplectic exceptional sphere in $\left(M_{0}, \omega_{0}\right)$. Then $(M, \omega)$ contains a symplectic exceptional sphere $C$ such that

$$
\int_{C} \omega=\int_{C_{0}} \omega_{0}
$$


In the proof of Lemma 3.1, Lemma A.13 can be replaced by a "soft" argument; see the arXiv version of this paper, [20, Lemma A.15 and Appendix B].

Sketch of proof of Lemma A.13. Suppose that $(M, \omega)$ is obtained from $\left(M_{0}, \omega_{0}\right)$ by a sequence of symplectic blow-ups: $M_{0}, M_{1}, \ldots, M_{s}=M$. Let $\Sigma_{0}$ be a symplectic exceptional sphere in $\left(M_{0}, \omega_{0}\right)$. We claim that there exists a symplectic exceptional sphere in $(M, \omega)$ in (the image in $H_{2}(M)$ of) the class $\left[\Sigma_{0}\right]$.

By induction, it is enough to address the case $s=1$ of a single blow-up. Let $\iota: B^{4}(\delta) \hookrightarrow M_{0}$ be a symplectic embedding defining the blow-up. By a parametrized version of the $C_{1}$ lemma, and because exceptional $J$-holomorphic spheres persist along any deformation of regular pairs $\left\{\left(\omega_{t}, J_{t}\right)\right\}$, see $[\mathbf{2 6}, \S 3]$, it is enough to show the claim for a deformation equivalent symplectic form on $M$. Since any two symplectic blow-ups centered at the same fixed point are deformation equivalent, this implies that we can choose the size $\delta$ arbitrarily small. If $\delta$ is small enough, we can find a Hamiltonian isotopy $\phi_{t}: M_{0} \rightarrow M_{0}, t \in[0,1]$, such that the image of $\phi_{1} \circ \iota$ is disjoint from $\Sigma_{0}$. Because the embeddings $\iota$ and $\phi_{1} \circ \iota$ are isotopic, the symplectic blow-up along $\phi_{1} \circ \iota$ gives a manifold $\left(M^{\prime}, \omega^{\prime}\right)$ symplectomorphic to $(M, \omega)$, see $[\mathbf{2 6}, \S 3]$. Because the symplectic blow-up operation takes place in an arbitrarily small neighborhood of the embedded closed ball, $\left(M^{\prime}, \omega^{\prime}\right)$, and thus $(M, \omega)$, contains an embedded symplectic sphere representing the class $\left[\Sigma_{0}\right]$.

Remark A.14. Symplectic exceptional spheres in four-manifolds can be studied using Taubes-Seiberg-Witten theory of Gromov invariants, see $[\mathbf{2 4}, \mathbf{2 9}]$. We can apply this theory to reduce the claim in Lemma A.13 on the existence of a symplectic exceptional sphere to a claim on the existence of an embedded smooth sphere in the corresponding homology class, and then deduce the latter claim from the construction of smooth blow-ups.

A.15. We now have holomorphic proof of Lemma 3.1. Let $(M, \omega)$ be a compact connected four-manifold. Let $\Delta$ be a moment map image for a toric action on $(M, \omega)$, and suppose that $\Delta$ can be obtained from a Delzant polygon $\Delta^{\prime}$ by a sequence of corner choppings of sizes $\delta_{1}, \ldots, \delta_{s}$. By $\S 3.2$,

$$
\delta_{i}<\frac{1}{2 \pi} K
$$

for all $i$, where

$$
K=2^{\operatorname{dim} H_{2}(M ; \mathbb{R})} \int_{M} \omega \wedge c_{1}(T M) .
$$

By assumption, there exist Delzant polytopes

$$
\Delta^{\prime}=\Delta_{0}, \Delta_{1}, \ldots, \Delta_{s}=\Delta
$$


such that each $\Delta_{i}$ is obtained from $\Delta_{i-1}$ by a corner chopping of size $\delta_{i}$. By Remark 2.12 and Delzant's theorem (see part (2) of Proposition 2.3), these correspond to a sequence of symplectic toric manifolds such that each manifold in the sequence can be obtained from the previous one by a blow-up of size $\delta_{i}$, and such that the last manifold in the sequence is $(M, \omega)$. Thus, $(M, \omega)$ can be obtained by a sequence of symplectic blow-ups from a symplectic manifold that contains a sphere of size $\delta_{i}$. By Lemma A.13, $(M, \omega)$ contains a symplectic exceptional sphere of size $\delta_{i}$. Thus, the $\delta_{i}$ 's belong to the set

$$
\begin{aligned}
\mathcal{D}(M, \omega):= & \left\{\frac{1}{2 \pi} \int_{C} \omega \mid C \subset M\right. \text { is a symplectic exceptional sphere } \\
& \text { and } \left.\int_{C} \omega \leq K\right\} .
\end{aligned}
$$

This set is finite, by Lemma A.1.

\section{References}

[1] M. Abreu and D. McDuff, Topology of symplectomorphism groups of rational ruled surfaces, J. Amer. Math. Soc. 13 (4) (2000), 971-1009 (electronic). MR1775741 (2001k:57035).

[2] S. Anjos and G. Granja, Homotopy decomposition of a group of symplectomorphisms of $S^{2} \times S^{2}$, Topology 43 (3) (2004), 599-618.

[3] M. Atiyah, Convexity and commuting hamiltonians, Bull. London Math. Soc. 14 (1982), 1-15.

[4] M. Audin, Torus actions on symplectic manifolds, 2nd revised ed., Progress in Mathematics, 93, Birkäuser-Verlag, Basel, 2004.

[5] P. Biran, Connectedness of spaces of symplectic embeddings, Inter. Math. Research Notices 1996, 10, 487-491. MR1399413 (97g:58022).

[6] A. Bloch, M. Ould El Hadrami, H. Flaschka and T.S. Ratiu, Maximal tori of some symplectomorphism groups and applications to convexity, in 'Deformation theory and symplectic geometry (Ascona, 1996),' Mathematical Physics Studies, 20, Kluwer Acad. Publ., Dordrecht, 1997, 201-222.

[7] O. Buse, Whitehead products in symplectomorphism groups and Gromov-Witten invariants, arXiv.math.SG/0411108.

[8] T. Delzant, Hamiltoniens périodiques et image convexe de l'application moment, Bulletin de la Société Mathématique de France 116 (1988), $315-339$.

[9] M. Demazure, Surfaces de del Pezzo II, in 'Séminaire sur les ingularités des Surfaces' (M. Demazure, H.C. Pinkham and B. Teissier, eds.), Lecture Notes in Mathematics, 777, Springer, Berlin, 1980, 23-35.

[10] W. Fulton, Introduction to toric varieties, Princeton University Press, 1993. 
[11] V. Ginzburg, V. Guillemin and Y. Karshon, Moment maps, Cobordisms, and Hamiltonian Group Actions, American Mathematical Society, Monographs, 98, 2002.

[12] R.E. Gompf, Some new symplectic 4-manifolds, Turkish J. Math. 18 (1) (1994), 7-15.

[13] M. Gromov, Pseudo holomorphic curves in symplectic manifolds, Invent. Math. 82 (1985), 307-347. MR0809718 (87j:53053).

[14] V. Guillemin and S. Sternberg, Convexity properties of the moment mapping, Invent. Math. 67 (1982), 491-513.

[15] V. Guillemin and S. Sternberg, Symplectic techniques in physics, Cambridge University Press, 1984.

[16] H. Hofer, V. Lizan and J.-C. Sikorav, On genericity for holomorphic curves in four-dimensional almost-complex manifolds, J. Geom. Anal. 7 (1) (1997), 149-159.

[17] Y. Karshon, Periodic Hamiltonian flows on four-dimensional manifolds, Memoirs of the American Mathematical Society 141 (672), (1999).

[18] Y. Karshon, Maximal tori in the symplectomorphism groups of Hirzebruch surfaces, Math. Res. Lett. 10 (1) (2003), 125-132.

[19] Y. Karshon and L. Kessler, Circle and torus actions on equal symplectic blow-ups of $\mathbb{C P}^{2}$, Math. Res. Lett., to appear, arXiv:math.SG/0501011.

[20] Y. Karshon, L. Kessler and M. Pinsonnault, A compact symplectic four-manifold admits only finitely many inequivalent toric actions, arXiv:math.SG/0609043.

[21] J. Kędra and D. McDuff, Homotopy properties of Hamiltonian group actions, Geom. Topol. 9 (2005), 121-162 (electronic). MR2115670 (2005j:53101).

[22] F. Lalonde and M. Pinsonnault, The topology of the space of symplectic balls in rational 4-manifolds, Duke Math. J. 122 (2) (2004), 347-397.

[23] E. Lerman, Contact cuts, Israel J. Math. 124 (2001), 77-92.

[24] T.J. Li, Smoothly embedded spheres in symplectic 4-manifolds, Proc. Amer. Math. Soc. 127 (2) (1999), 609-613. MR1459135 (99c:57055).

[25] Yu.I. Manin, Cubic forms: algebra, geometry, arithmetic (Translated by M. Hazewinkel), North-Holland Publishing Co., Amsterdam, London, 1974.

[26] D. McDuff, The structure of rational and ruled symplectic 4-manifolds, J. Amer. Math. Soc. 3 (3) (1990), 679-712. MR1049697.

[27] D. McDuff, Blow ups and symplectic embeddings in dimension 4, Topology 30 (3) (1991), 409-421.

[28] D. McDuff, Singularities and positivity of intersections of $J$ holomorphic curves, in 'Holomorphic curves in symplectic geometry,' Chapter VI (M. Audin and J. Lafontaine, Eds.), Progress in Mathematics 117, Birkhäuser, 1994. 
[29] D. McDuff, From symplectic deformation to isotopy, in Topics in symplectic 4-manifolds (Irvine, CA, 1996), 85-99, First Int. Press Lect. Ser., I, Internat. Press, Cambridge, MA, 1998. MR1635697.

[30] D. McDuff, A survey of the topological properties of symplectomorphism groups, arXiv math.SG/0404340.

[31] D. McDuff and D. Salamon, Introduction to symplectic topology, 2nd ed., Oxford University Press, 1998.

[32] D. McDuff and D. Salamon, J-holomorphic curves and symplectic topology, Amer. Math. Soc., 2004.

[33] M. Pinsonnault, Maximal tori in the groups of Hamiltonian automorphisms of symplectic 4-manifolds, arXiv:math.SG/0612565.

[34] A.G. Reznikov, Characteristic classes in symplectic topology, Selecta Math. (N.S.) 3 (4) (1997), 601-642.

[35] P. Seidel, Floer homology and the symplectic isotopy problem, Ph.D. thesis, Oxford University, 1997.

[36] J.-C. Sikorav, Some properties of holomorphic curves in almost complex manifolds, in 'Holomorphic curves in symplectic geometry,' Chapter V (M. Audin, J. Lafontaine, eds.), Progress in Mathematics, 117, Birkhäuser, 1994.

[37] L. Traynor, Symplectic packing constructions, J. Diff. Geom. 42 (2) (1995), 411-429.

[38] A. Weinstein, Symplectic manifolds and their Lagrangian submanifolds, Adv. Math. 6 (1971), 329-346.

Department of Mathematics

UNIVERSITY OF TORONTO

TORONTO

ONTARIO

CANADA M5S 2E4

E-mail address: karshon@math.toronto.edu

Courant Institute of Mathematical Sciences

NeW YORK UNIVERSITY

NEW YORK

NY 10012

E-mail address: kessler@cims.nyu.edu

Department of Mathematics

University OF TORONTO

TORONTO

ONTARIO

CANADA M5S 2E4

Current Address:

Fields Institute

TORONTO

ONTARIO

CANADA M5T 3J1

E-mail address: mpinsonn@fields.utoronto.ca 
Received 9/1/2006, accepted 7/17/2007

We are grateful to Paul Biran, Dietmar Salamon, and Margaret Symington for helpful conversations. The project is partially funded by an NSERC Discovery grant, an NSERC LSI grant, an NSERC postdoctoral grant number BP-301203-2004, and a VIGRE grant DMS-9983190. 